\title{
Evaluación in vitro de la rugosidad superficial y la alteración de color de dos tipos de ionómeros de vidrio, luego de ser sometidos a diferentes bebidas
}

\section{Resumen}

El objetivo de este estudio in vitro fue analizar la rugosidad superficial y la alteración de color de dos tipos de ionómeros vítreos luego de ser sometidos a diferentes soluciones. En una matriz de teflón $(8 \times 2 \mathrm{~mm})$, fueron confeccionados 60 cuerpos de prueba con 2 tipos de ionómeros fotopolimerizables: 30 para el Fuji II LC (M1) y otros 30 para el Ketac N100 (M2). El aparato utilizado fue el Elipar Freelight $23 \mathrm{M}$ Espe. La fotopolimerización se realizó por 20 segundos cada cuerpo de prueba. Luego de la confección, los sesenta cuerpos de prueba fueron mantenidos en gasa humedecida por 24 hs en estufa a $37^{\circ} \mathrm{C}$ y luego se clasificaron y luego se dividieron en tres grupos de 10 cada uno para ser sometidos a 3 tipos diferentes de soluciones: agua destilada (S1), bebida carbonatada (S2) y jugo cítrico (S3) por 90 segundos diariamente
Marisol Carrillo Tabakman ${ }^{1}$, David E Ugarte Nuñez², Alma Blasida Concepcion Elizaur Benitez Catirse',

Paulo Nelson Filho ${ }^{3}$ durante 14 días. Las lecturas de la alteración de color, obtenidas a través de un colorímetro, y las de rugosidad superficial, realizadas por medio del rugosímetro, fueron realizadas a las 48 hs. (T0) y luego a los 14 días (T1). Los resultados obtenidos fueron sometidos al test ANOVA y Tukey $(\mathrm{p} \leq 0.05)$. Los resultados mostraron que; a) La bebida carbonatada (S2) tuvo mayor media de alteración de color $\left(\Delta \mathrm{E}^{*}\right)$ en relación a las otras soluciones, b) que Ketac N100 (M2) tuvo mayor media con respecto a la rugosidad superficial en la interacción material por solución. Por tanto, se concluyó que trascurrido determinado período de tiempo las propiedades estéticas y físico-mecánicas de los materiales estudiados se ven afectados.

Palabras Clave: ionómeros vítreos, nano ionómeros, rugosidad superficial, alteración de color, bebidas carbonatadas, jugo cítrico.

1. DDS, MSc, Practica Privada, Asunción, Paraguay - mscarrillod@hotmail.com Telefono: 595971202311.

2. DDS, MSc , PHD, Vice Decano de la Facultad de Odontología Universidad Nacional de Concepción.

3. DDS, MSc , PHD, Departamento de Prostodoncia y Materiales dentales - Facultad de Odontología de Riberao Preto- Universidad de Sao Paulo.

4. DDS, MSc, PHD, Profesor de Odontopediatría, Facultad de Odontología de Riberao Preto, Universidad de Sao Paulo. 
Artigo Original

\section{Avaliação in vitro da rugosidade superficial e alteração de cor de dois tipos de ionômeros de vidro, depois de ter sido submetido a bebidas diferentes}

\section{Resumo}

O objetivo deste estudo in vitro foi analisar a rugosidade superficial e a alteração de cor de dois tipos de ionômeros de vidrio após serem submetidos a diferentes bebidas. Em uma matriz de te ón $(8 \times 2 \mathrm{~mm})$, foram confeccionados 60 corpos de prova com 2 tipos de ionômeros fotopolimerizáveis: 30 para o Fuji II LC (M1) e outros 30 para o Ketac N100 (M2). O aparelho utilizado foi o Elipar Freelight 2 3M Espe. A fotopolimerização foi realizada por 20 segundos em cada corpo de prova. Após a confecção, os 60 corpos de prova foram mantidos em gaze umedecida por $24 \mathrm{hrs}$ em estufa a 37o C e divididos em 3 grupos de 10 cada um, para ser submetidos a 3 tipos diferentes de soluções: água destilada (S1), bebida carbonatada (S2) e suco cítrico (S3) por 90 segundos diariamen- te durante 14 dias. As leituras de alteração de cor, obtidas através de um colorímetro, e das de rugosidades superficial, realizadas por meio do rugosímetro, foram realizadas após 48 hs. (T0) e após 14 dias (T1). Os resultados obtidos foram submetidos à test ANOVA e Tukey ( $\mathrm{p} \leq 0.05)$. Os resultados mostraram que; a) a bebida carbonatada (S2) teve maior media de alteração de cor $\left(\Delta \mathrm{E}^{*}\right)$ em relação às outras soluções, b) que Ketac N100 (M2) teve maior media com respeito a rugosidade superficial na interação material pela solução. Podemos concluir que a partir de determinado período de tempo as propriedades estéticas e físico-mecânicas dos materiais estudados são afetadas.

Palavras-chave: ionômeros de vidrio, nanoionômeros, rugosidade superficial, estabilidade da cor, bebidas carbonatadas, suco cítrico.

Original article

\section{In vitro evaluation of surface roughness and color alteration of two types of glass ionomers, after being subjected to different beverages}

\section{Summary}

The aim of this in vitro study was to examine the surface roughness and discoloration of two types of glass ionomer had been subjected to different solutions. In a matrix of Teflon $(8 \times 2 \mathrm{~mm}), 60$ test bodies were prepared with 2 types of resin modified glass ionomers: 30 for Fuji II LC ${ }^{\oplus}$ (M1) and the other 30

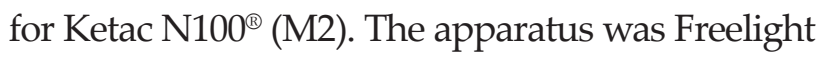
two 3M ESPE Elipar. Photopolymerization was carried out for 20 second each test body. After the preparation, the seventy-two specimens were kept in gauze soaked for 24 hours in an oven at $37^{\circ} \mathrm{C}$ and then were sorted and divided into three groups of 12 each to be subjected, for 90 seconds each day at 3 different types of solutions: distilled water (S1), 
carbonated beverage (S2) and citrus juice (S3) for 14 days. Readings from discoloration, obtained through a colorimeter, and the surface roughness made using the profilometer were performed after 48 hours (T0) and after day 14 (T1). The results were submitted to ANOVA and Tukey test $(\mathrm{p} \leq 0.05)$. The results showed that: a) The carbonated beverage (S2) had higher mean discoloration $\left(\Delta \mathrm{E}^{*}\right)$ compared to the other solutions, b) Ketac N100®(M2)

\section{Introducción}

En las últimas décadas, ha aumentado considerablemente la necesidad de encontrar materiales más estéticos y biocompatibles no solo en adultos sino también en niños. ${ }^{1}$

Los cementos de ionómeros vítreos han sido ampliamente utilizados en la Odontología restauradora debido a sus ventajosas propiedades como son la biocompatibilidad, la adhesión a la estructura dental y la liberación de flúor. ${ }^{2}$ Estudios laboratoriales han demostrado claramente el gran efecto cariostático que poseen los ionómeros en el desarrollo y avance de la lesión cariosa. La superficie del esmalte y la dentina adyacente a la obturación con cementos de ionómeros vítreos son resistentes al ataque ácido, lo que indica que pueden inhibir la desmineralización causada por el ataque ácido a la estructura dental. ${ }^{3}$ La liberación de flúor de los materiales dentales fue ampliamente estudiada. ${ }^{4}$ Esta propiedad es una de las características más importantes de los cementos de ionomeros vítreos y no ha sido superada por ningún otro material. Estos estudios han comprobado que los iones $\mathrm{F}^{-}$contenidos en el material pueden, fortalecer la estructura dental, promover la remineralización e inhibir, así, la desmineralización dental. had higher mean with respect to the interaction surface roughness material solution. Therefore, it was concluded that certain period of time elapsed aesthetic properties and physico-mechanical properties of the material studied are affected.

Keywords: glass ionomer, nano ionomer, surface roughness, color stability, cola beverage, orange juice.

Los cementos de ionómeros vítreos, así como los modificados con resinas compuestas, los compómeros y las resinas compuestas modificadas con poliácidos han recibido especial atención debido a esta propiedad de liberación de flúor que puede ser incorporada al mineral dentinario y formar fluorapatita o hidroxiapatita F-enriquecido, ambos reducen la solubilidad del tejido dentario, como consecuencia lo hace más resistente al ataque ácido. 5,6

La adhesión del material a las paredes cavitarias de la restauración es una de las propiedades más importantes que debe presentar el material ideal pues esto previene la microfiltración. La microfiltración es definida como el pasaje químicamente indetectable de bacterias, fluídos, moléculas o iones entre la restauración dentaria y las paredes cavitarias.

Los cementos de ionómero de vidrio son biomateriales muy eficaces por su adhesión al tejido dentinario. Clínicamente, el enlace ionómero de vidrio-diente parece resistir muy bien al ambiente agresivo de la cavidad oral. ${ }^{7}$

En una revisión bibliográfica, el mejor resultado entre cinco mecanismos diferentes de adhesión clínica se ha documentado con los cementos de 
ionómeros vítreos. Su porcentaje promedio anual fracaso fue significativamente menor que la de los otros sistemas adhesivos también evaluados. ${ }^{8}$

Sin embargo, estos materiales tienen algunas limitaciones clínicas tales como la sensibilidad a la humedad durante su aplicación, el prolongado tiempo de fraguado que dificulta la terminación y el pulido, la deshidratación del mismo y la baja textura superficial, lo que disminuye la resistencia mecánica del material. Las bajas propiedades mecánicas de estos materiales hacen que sean de uso limitado. ${ }^{9}$

Estas limitaciones han sido superadas por la introducción de las resinas modificadas-cementos de ionómero vítreos. Dentro del campo de las resinas modificadas-cementos de ionómero vítreos han sido desarrollados los nanoionómeros, que han combinado el beneficio de los cementos ionómeros con resina modificada junto a la tecnología del nanorelleno. Hoy día, existen una gran variedad de tipos de cementos de ionómeros vítreos disponibles en el mercado.

La nanotecnología, también conocida como de ingeniería molecular o nanotecnología molecular, es la producción funcional de materiales y estructuras dentro de un intervalo de 0,1 a 100 nanómetros - la nano escala - por varios métodos físicos o químicos. El gran interés en su uso, parte de la idea que puedan ser utilizados para modificar la estructura de los materiales y mejorar las propiedades eléctricas, químicas, mecánicas y ópticas. ${ }^{10}$

En los últimos años, la nanotecnología ha sido utilizada en diferentes tipos de resinas compuestas. Recientemente, ha sido introducido al mercado, un cemento de ionómero vítreo modificado con resina basada en la nanotecnología el KetacN100® - 3M-ESPE, el cual posee propieda- des mejoradas en cuanto a la textura superficial y la estética. ${ }^{11}$

Los nano rellenos incorporados a los cementos de ionómeros vítreos mejoran aparentemente sus propiedades mecánicas. ${ }^{12}$

Recientes estudios experimentales in vitro han conducido a observar la influencia de soluciones fluoradas en la rugosidad superficial y la dureza de las restauraciones luego de sumergirlas en las mismas. ${ }^{13}$

También se observaron cambios en la estabilidad de color de los materiales estéticos, principalmente en resinas compuestas, luego de ser sumergidos en bebidas gaseosas y otros líquidos como el café y el vino. ${ }^{14}$ Pero básicamente han sido poco estudiados aún, los cementos ionoméricos, sumergidos en líquidos habitualmente consumidos por los niños, como podríamos citar las bebidas carbonatadas y los jugos cítricos. ${ }^{15}$ Por tanto, estos análisis aún son limitados.

Además podemos agregar que, los estudios comparativos existentes, en cuanto a beneficios $\mathrm{y}$ limitaciones de un $\mathrm{u}$ otro tipo de cementos ionómeros vítreos indicado para cada caso son todavía escasos. 16 Así también, las evaluaciones comparativas de las diferentes propiedades mecánicas han sido aún poco estudiadas; por lo cual, el propósito de este trabajo será evaluar la rugosidad superficial y la alteración de color de dos tipos diferentes de cementos de ionómeros vítreos modificados sometidos a diferentes soluciones, de manera a determinar el grado de alteración en dichas propiedades que éstos producen en dichos materiales, para ampliar y difundir los conocimientos con base científica.

El objetivo de este estudio fue evaluar, in vitro, la alteración de color y la rugosidad superficial 


\begin{tabular}{|c|l|c|c|}
\hline Material & \multicolumn{1}{|c|}{ Composición } & $\begin{array}{c}\text { Tiempo de } \\
\text { fotopolimerización }\end{array}$ & Fabricante \\
\hline Fuji II LC & $\begin{array}{l}\text { Polvo: Vidrio fluoro- } \\
\text { aluminio- silicato. } \\
\text { Líquido: Acido poliacrílico } \\
\text {-2Hidroxietil metacrilato } \\
\text { (HEMA)- Dimetacrilato- } \\
\text { Canforquinona- Agua }\end{array}$ & 20 seg. & $\begin{array}{c}\text { GC Corporation } \\
\text { Tokyo, Japan }\end{array}$ \\
\hline Ketac N100 & $\begin{array}{l}\text { Agua destilada, HEMA, } \\
\text { Copolimero del Vitrebond } \\
\text { (metacrilato modificado áci- } \\
\text { do polialquenoico), Vidrio } \\
\text { de Fluoroaluminosilicato, } \\
\text { nanómeros yrupos nanos. }\end{array}$ & 20 seg. & $\begin{array}{c}\text { Dental Products, St. Paul, } \\
\text { MN, USA. }\end{array}$ \\
\hline
\end{tabular}

Tabla 1. Características de los materiales de restauración empleados.

de dos tipos de cementos de ionómeros vítreos modificados luego de ser sumergidos a tres diferentes tipos de bebidas.

\section{MATERIAL Y MÉTODOS.}

Materiales, instrumentales, aparatos y equipos auxiliares.

Para el desarrollo experimental de este estudio fueron utilizados dos tipos de cementos de ionómeros; uno, con resina modificada y el otro un nanoionómero, ambos de color A2 (Tabla 1) y tres tipos de soluciones (Tabla2), conforme a las especificaciones contenidas en sus respectivos cuadros.
- Matriz de teflón (2x6 mm).

- Aparato de fotopolimerización Elipar (3M-ESPE).

- Estufa y

- Discos de pulir Super Snap (Shofu).

\section{Aparatos para las lecturas.}

Para las lecturas de color fue utilizado el COLORIMETRO - modelo Color Guide 45/0, BYK-Gardner (Gerestreid, Alemania), y las lecturas de rugosidad superficial fueron obtenidas con el RUGOSIMETRO - modelo SJ-201 P/M (Mitutoyo, Tokyo, Japón) - Aparatos del Departamento de Materiales Dentales de la Facultad de Odontología de Ribeirão Preto de la Universidad de San Pablo .

\section{Métodos.}

Para la confección de los cuerpos de prueba fueron utilizados los siguientes materiales:

- Espátula de teflón.

\section{Delineamiento experimental.}

Para evaluar in vitro la influencia de las soluciones cítricas y carbonatadas en la alteración 


\begin{tabular}{|c|c|c|}
\hline Tipo & Marca & Fabricante \\
\hline Agua destilada & Nelschel & Asunción - Paraguay \\
\hline Bebida Carbonatada & Coca Cola ${ }^{\bullet}$ & Coca Cola $^{\star}$ Paraguay \\
\hline Jugo Cítrico & Frugos & Coca Cola - Paraguay \\
\hline
\end{tabular}

Tabla 2. Características de las soluciones utilizadas.

del color y la rugosidad superficial sobre dos diferentes tipos de cementos de ionómeros vítreos fue realizado un estudio piloto que permitió determinar los niveles de los factores en estudio, padronizar la técnica de confección de los especímenes, establecer las condiciones del estudio y controlar los factores que podrían interferir en el análisis. Los factores en estudio están descriptos con sus respectivas siglas y diferentes niveles, a seguir:
A. Factor Material (M) - Cementos de ionómeros de vidrio - color A2 con 2 niveles:

M1- Fuji II LC.

M2- Ketac N100.

B. Factor Solución (S) - con 3 niveles:

S1- Agua destilada (control)

S2- Bebida Carbonatada.

S3- Desafío Cítrico.

C. Factor Tiempo $(\mathrm{T})-$ con 2 niveles:

T0- Tiempo inicial.

$\mathrm{T} 1$ - A los 14 días.

\section{Delineamiento Estadístico}

En el plan piloto también fue determinado el número de cuerpos de prueba para cada condición experimental y el número de lecturas para las va- riables en estudio. Se adoptó el delineamiento experimental según el modelo factorial 2x3×2.

La muestra del experimento tuvo 60 cuerpos de prueba, hechos 30 con Fuji II LC y 30 con Ketac N100, siendo $n=10$ para cada condición experimental. Para la variable - alteración de color - y los parámetros $\mathrm{L}^{*}, \mathrm{a}^{*} \mathrm{y} \mathrm{b}^{*}$ fueron establecidas un total de 120 lecturas. Para la variable - rugosidad superficial - fue establecida la realización de tres lecturas para cada cuerpo de prueba, en el tiempo inicial (T0) y el tiempo final (T1) totalizando un número de 360 lecturas.

Las variables de respuestas continúas fueron:

1 - Alteración de color $\left(\Delta \mathrm{E}^{*}\right)$

2 - Rugosidad superficial (Ra)

Fue determinada la realización de los análisis estadísticos, por medio de la aplicación de Adherencia a Curva Normal, lo que definió la utilización del test paramétrico ANOVA $(p<0.05)$ y el test complementario de Tukey. El nivel de significancia adoptado fue 5\%. Para este análisis estadístico se utilizo el programa estadístico GMC - versión 2002.

\section{Primera fase del experimento Obtención de los cuerpos de prueba.}

Para la confección de los cuerpos de prueba fueron empleados los cementos de ionómeros vítreos con 
resina modificada Fuji II LC y el Ketac N100 de color A2 cuyas especificaciones fueron descriptas en el Cuadro 1.

Los cuerpos de prueba fueron confeccionados y obtenidos con una matriz de teflón de 8 milímetros de diámetro y 2 milímetros de espesor. Los cementos de ionómeros vítreos fueron incorporados en la matriz en un único incremento con jeringa de inserción (3M-Espe). Luego de la inserción del material, fue colocada una tira de celuloide y encima una loseta de vidrio fue colocada sobre la matriz de teflón con una carga de 500 gramos durante 30 segundos para garantizar que toda la matriz fuese llenada y permitir también así la eliminación del exceso de material. Luego de la remoción de la carga se realizó la fotopolimerización del material con el aparato de fotopolimerización Elipar 3M-Espe de acuerdo a las indicaciones del fabricante (Cuadro 1). Antes de realizar cada fotopolimerización, la potencia del aparato $\left(600 \mathrm{~mW} / \mathrm{cm}^{2}\right)$ fue testeada mediante el uso del radiómetro - Demetron Reseach Corp.

Luego de realizar la fotopolimerización, los cuerpos de prueba fueron retirados de la matriz y mantenidos en contenedores con gasa humedecida con agua destilada durante 24 horas en estufa a una temperatura de $37^{\circ} \mathrm{C} \pm 1^{\circ} \mathrm{C}$, para luego de este tiempo ser pulidos con los discos Super Snap (Shofu®). Primero con los discos de grano grueso y luego con discos de grano fino, humedeciendo la superficie intercaladamente para que no se produzca un resecamiento y posteriormente el resquebrajamiento de la superficie, lo que, consecuentemente alteraría la superficie a ser evaluada.

La cara del espécimen o cuerpo de prueba que ha sido pulida se ha marcado debidamente ya que luego servirá de guía para su posicionamiento en el aparato, tanto en el rugosímetro como en el colorímetro espectrofotométrico.

\section{Almacenamiento de los cuerpos de prueba}

Los cuerpos de prueba fueron almacenados en recipientes plásticos debidamente identificados conteniendo gasa humedecida con $5 \mathrm{ml}$. de agua destilada cada recipiente, en estufa a una temperatura de $37^{\circ} \mathrm{C} \pm 1^{\circ} \mathrm{C}$, hasta el momento de ser sometidos a las lecturas iniciales, tanto para rugosidad superficial como para color, para luego realizar la inmersión en las diferentes soluciones propuestas.

\section{Lecturas.}

\section{Lecturas de Alteración de Color.}

Las lecturas del color fueron realizadas inmediatamente luego de 24 horas del término del período experimental. Para la evaluación del color fue empleado el espectofotómetro PCB 6807 BYK Gardner - modelo Color Guide 45/0, (Gerestsreid, Alemania), con abertura focal de $11 \mathrm{~mm}$ y la geometría óptica utilizada fue de D65 de iluminación y el ángulo de observación de $10^{\circ}$ (REIS, 2003). Con una pinza clínica fue manipulado cada cuerpo de prueba, de manera a evitar el contacto con la mano del evaluador, ya que esto podría depositar residuos o grasa y de esta forma alterar o influenciar los valores encontrados. Los cuerpos de prueba fueron también secos con papel absorbente para impedir la interferencia de la humedad en los valores de la lectura.

El aparato emite una fuente de luz con ondas de entre 400 a $700 \mathrm{~nm}$ sobre el objeto y mide la reflexión de este espectro. La geometría de medición utilizada fue de 45/0, lo que significa, que el proceso se establece a una iluminación circular de $45^{\circ} \mathrm{y}$ 
se observa a un ángulo de $0^{\circ}$. El aparato de medición utiliza dos electrominescentes de alta duración para iluminar la muestra.

Para los objetos translúcidos, el valor del color del plano de fondo es adicionado al valor del color del objeto, siendo así los cuerpos de prueba colocados sobre un bloque de fondo de color blanco y opaco (Standard For 45으, $0^{\circ}$ Reflectance and Color Gardner Laboratory Inc. Bethesda, Maryland 20014). (Reis, 2003).

Las medidas del color fueron obtenidas por el sistema de color CIE L* $a^{*} b^{*}$. La diferencia total entre dos estímulos de color $\left(\Delta \mathrm{E}^{*}\right)$ fue calculada automáticamente por la siguiente fórmula:

\section{$\Delta \mathrm{E}^{*}=$}

El sistema CIE L*a*b* utiliza tres parámetros para definir color; estos son: luminosidad, matiz y saturación (COMISSION INTERNATIONALE DE L'ECLARE, 1978). La luminosidad representa el grado de claro u obscuro de un objeto y está representado por el valor $L^{*}$, siendo $L^{*}=100$ para blanco y la $L^{*}=0$ para negro. Los parámetros de $a^{*} \mathrm{y} \mathrm{b}^{*}$, llamados escala cromática (matiz), representan el rojo si es $+a^{*}$ y el verde si fuese $-a^{*}$, el amarillo en cuanto sea $+b^{*}$ y el azul si es $-b^{*}$ (Schulze et al, 2003). La saturación y la intensidad del matiz es dado por el valor numérico de $\mathrm{a}^{*} \mathrm{y} \mathrm{b}^{*}$. Los valores de $\Delta \mathrm{L}^{*}, \Delta \mathrm{a}^{*}, \Delta \mathrm{b}^{*}$ corresponden a la diferencia de los valores de $L^{*}, a^{*}, b^{*}$, respectivamente, en comparación a la primera lectura de color o inicial. (Figura 1).

Este sistema consiste en dos ejes $\mathrm{a}^{*} \mathrm{y} \mathrm{b}^{*}$ que forman ángulos rectos y representan la dimensión de la tonalidad o color. El tercer eje es la luminosidad $L^{*}$. Este es perpendicular al plano $a^{*} b^{*}$. Con este sistema cualquier color puede ser especificado con

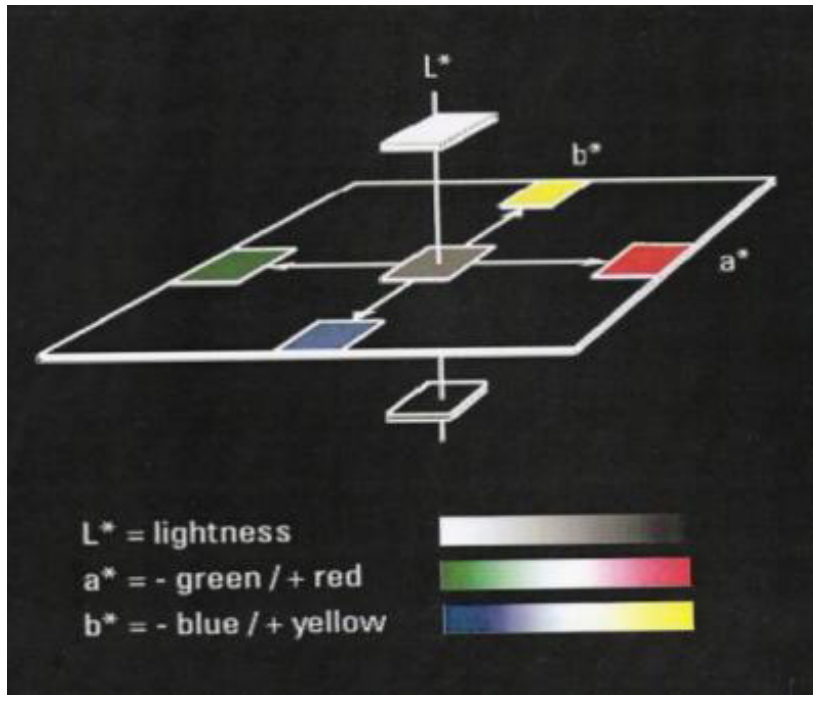

Figura 1. Representación gráfica del sistema CIE $L^{*} a^{*} b^{*}$.

las coordenadas $L^{*}, a^{*}, b^{*}$. El cambio de color, $\Delta E^{*}$, es comúnmente usada para representar una diferencia de color (Freitas, 2006).

\section{Lectura de Rugosidad Superficial}

Para las lecturas de rugosidad superficial fue utilizado el rugosímetro Mitutoyo SJ-201P (Tokio - Japón). Para la obtención de la lectura de rugosidad, la aguja del rugosímetro recorre una distancia de 2, $0 \mathrm{~mm}$ (meter cutt-off) en cada medida sobre el cuerpo de prueba con una velocidad de $0,5 \mathrm{~mm} / \mathrm{s}$.

$\mathrm{Al}$ ser accionado el aparato, la aguja realiza el recorrido en una única dirección, detectando así las irregularidades de la superficie medida. Esas irregularidades corresponden a picos, valles y ondas. De esta manera, se obtiene un valor que constituye la rugosidad media (Ra) de las superficies.

Para la realización de las lecturas, los especímenes tuvieron una de sus facetas marcadas y esto servía como guía para las lecturas, para que la misma fuese realizada en la misma cara en los dos momentos de la lectura, así como también para el 
posicionamiento de espécimen en el rugosímetro para leerlo.

En cada cuerpo de prueba se realizaron tres lecturas en lugares diferentes de la superficie de la muestra. Luego de las tres lecturas, los valores medios de la rugosidad de superficie fueron calculados.

\section{Segunda fase del experimento.}

Luego, los cuerpos de prueba fueron sumergidos en las distintas soluciones (S1, S2 y S3) por 14 días. Las bebidas fueron cambiadas cada 24 horas. Los cuerpos de prueba eran sumergidos cada día durante 90 segundos.

Luego de 14 días, los mismos procedimientos realizados en la primera fase fueron repetidos con cada uno de los cuerpos de prueba y todas las lecturas fueron hechas como se ha descrito previamente.

\section{RESULTADOS}

Los datos de las variables Alteración de color (Delta E) y Rugosidad superficial fueron sometidos al test de adherencia a la curva normal y siendo la distribución de la muestra normal, para los datos de ambos ensayos. Así, se realizó el análisis de Varianza para las dos variables.

\section{ALTERACIÓN DE COLOR}

El análisis de la Tabla 3 de varianza, obtenido por el Test ANOVA, se verifica que el factor Solución y la Interacción Solución x Material tuvieron efecto estadísticamente significante $(\mathrm{p} \leq 0,01)$ sobre la variable de estudio, la alteración de color. Con el objetivo de identificación de las diferencias de comportamiento fue utilizado el Test complementar de Tukey. El factor Material no tuvo efecto estadísticamente significante.

\begin{tabular}{|l|c|c|c|c|c|}
\hline \multicolumn{1}{|c|}{ Fonte de Variaç } & $\begin{array}{c}\text { Soma de } \\
\text { Quadr. }\end{array}$ & G.L. & Quadr.Médios & ( F ) & Prob.(H0) \\
\hline Factor SOLUCIÓN (S) & 11.6337 & 2 & 5.8169 & 11.39 & $0.020 \%$ \\
\hline Factor MATERIAL (M & 0.0073 & 1 & 0.0073 & 0.01 & $9.876 \%$ \\
\hline Interacción SxM & 46.8072 & 2 & 23.4036 & 45.83 & $0.000 \%$ \\
\hline Resíduo & 27.5780 & 54 & 0.5107 & & \\
\hline Variaçäo total & 86.0262 & 59 & & & \\
\hline * $\leq 0,01$ & & & & & \\
\hline
\end{tabular}

Tabla 3. Análisis de varianza: Valores originales de alteración de color $(\Delta E)$ 


\begin{tabular}{|l|l|l|}
\hline \multicolumn{1}{|c|}{ S1 } & \multicolumn{1}{c|}{ S2 } & \multicolumn{1}{c|}{ S3 } \\
\hline Agua destilada & $\begin{array}{l}\text { Bebida Carbo- } \\
\text { natada }\end{array}$ & Desafío Cítrico \\
\hline $1,86 \pm 0,15 \bullet$ & $2,53 \pm 0,15 \bullet$ & $1,51 \pm 0,15 \bullet$ \\
\hline $\begin{array}{l}\text { Valor crítico de } \\
\text { Tukey: } 0,83\end{array}$ & & \\
\hline Dp. $\pm 0,15$ & & \\
\hline
\end{tabular}

Tabla 4. Medias de Alteración de color $(\Delta E)$ para el Factor Solución.

Esto significa que:

a) Factor Solución.

El efecto estadísticamente significante del factor Solución, puede verificarse por medio de la diferencia entre las medias de alteración de color entre las soluciones estudiadas. En la Tabla 4 de medias y en el Gráfico 1, se observa que la Bebida Carbonatada (S2) presentó mayor media que las Soluciones: Agua destilada (S1) y el Desafío Cítrico (S3), que a su vez fueron estadísticamente iguales entre sí.

\section{b) Factor Material .}

El factor Material no tuvo efecto significativo sobre la alteración de color. Las medias con las cuales se confeccionó el Gráfico 2, están agrupadas en la Tabla 5 a seguir.

\section{c) Interacción Material x Solución}

La importancia de esta interacción está en la significancia estadística verificada. Esto principalmente porque cuando fue analizado el factor Material aisladamente, no tuvo efecto significante sobre la alteración de color. Para una mejor comprensión, el análisis de la Tabla 6, en el sentido de las líneas, se puede observar que para el Material Fuji II LC® (M1), la Bebida carbonatada (S2) determinó menor media de alteración de color, que cuando fue sometido a la acción del Agua destilada (S1), y una media es-

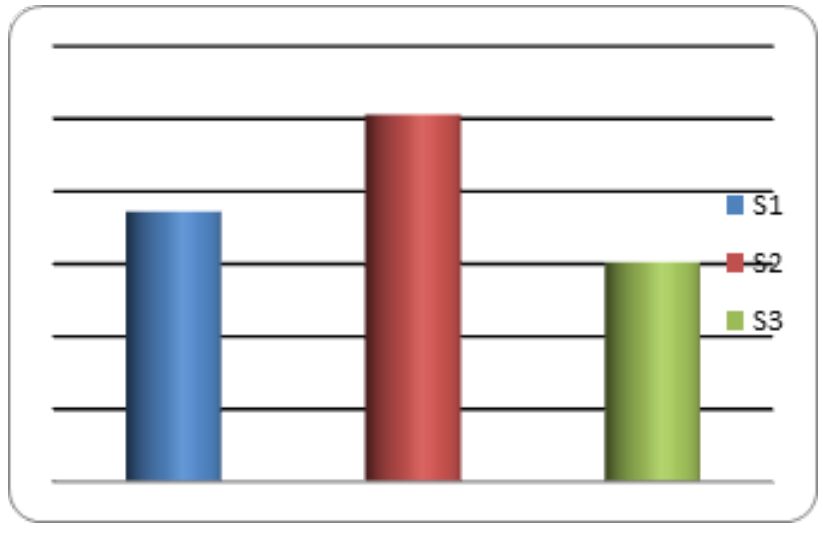

Gráfico 1. Medias de $\Delta E$ para el factor Solución.

tadísticamente igual que la media que presentó el Desafío cítrico (S3). La media de alteración de color promovida por la Solución S2 (Bebida Carbonatada) para el Material KetacN100® (M2), fue la mayor que las determinadas por S1 (Agua destilada) y S3 (Desafío Cítrico), que a su vez fueron estadísticamente iguales entre sí.

Realizándose las comparaciones en el sentido de las columnas, se verifica que el Agua destilada (S1) promovió menor media de alteración de color para el Material KetacN100® (M2) que Fuji II LC® (M1); la Bebida carbonatada (S2), promovió mayor media para el Material KetacN100® que para Fuji II LC®. Y la Bebida S3 (Desafío cítrico) promovió medias estadísticamente iguales entre sí, para los dos materiales estudiados. Esto puede ser mejor visto en los Gráficos 3 y 4, a seguir.

\section{c) Interacción Material x Solución}

La importancia de esta interacción está en la significancia estadística verificada. Esto princi-

\begin{tabular}{|c|c|}
\hline M1- Fuji II LC & M2-Ketac $^{\infty} \mathbf{N 1 0}^{\circ}$ \\
\hline 1,89 & 2,05 \\
\hline $\mathrm{dp} \pm 0,13$ & \\
\hline
\end{tabular}

Tabla 5. Medias de Alteración de color $(\Delta E)$ para el Factor Material. 


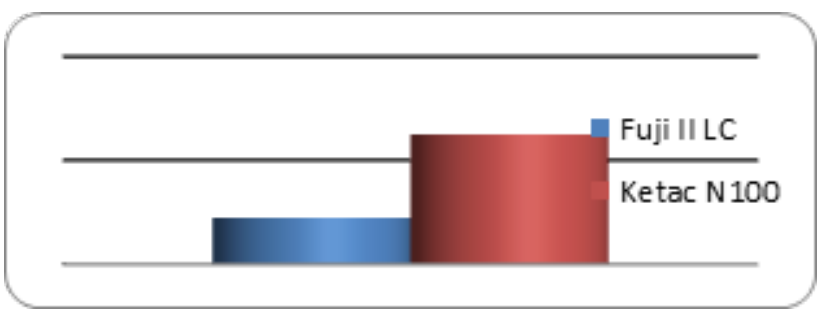

Gráfico 2. Medias de $\Delta$ E para el factor Material.

palmente porque cuando fue analizado el factor Material aisladamente, no tuvo efecto significante sobre la alteración de color. Para una mejor comprensión, el análisis de la Tabla 6, en el sentido de las líneas, se puede observar que para el Material Fuji II LC® (M1), la Bebida carbonatada (S2) determinó menor media de alteración de color, que cuando fue sometido a la acción del Agua destilada (S1), y una media estadísticamente igual que la media que presentó el Desafío cítrico (S3). La media de alteración de color promovida por la Solución S2 (Bebida Carbonatada) para el Material KetacN100@ (M2), fue la mayor que las determinadas por S1 (Agua destilada) y S3 (Desafío Cítrico), que a su vez fueron estadísticamente iguales entre sí.

Realizándose las comparaciones en el sentido de las columnas, se verifica que el Agua destilada (S1) promovió menor media de alteración de color para el Material KetacN100® (M2) que Fuji II LC® (M1); la Bebida carbonatada (S2), promovió mayor media para el Material KetacN100® que para Fuji II LC®. Y la Bebida

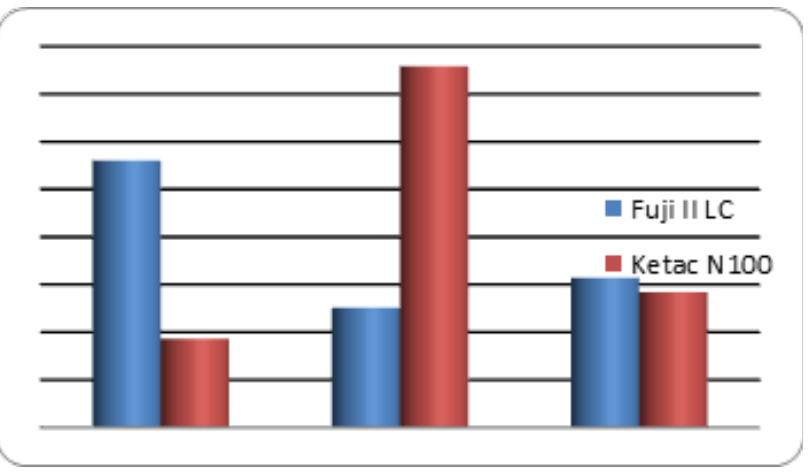

Gráfico 3. Medias de $\Delta E$ para la Interacción Solución $x$ Material (Sentido de las columnas).

S3 (Desafío cítrico) promovió medias estadísticamente iguales entre sí, para los dos materiales estudiados. Esto puede ser mejor visto en los Gráficos 3 y 4, a seguir.

\section{Rugosidad Superficial}

Para el análisis estadístico de los datos de Rugosidad superficial ( $\mathrm{Ra}$ ) se aplicó el Test de Adherencia a la Curva Normal que demostró que la muestra fue normal para la variable Rugosidad Superficial, siendo así fue utilizado luego el test paramétrico ANOVA.

\section{Factor Solución.}

El factor Solución no tuvo un efecto estadísticamente significativo sobre la rugosidad superfi-

\begin{tabular}{|l|c|c|c|}
\hline & S1 & S2 & S3 \\
& Agua Destilada & Bebida Carbonatada & Desafío Cítrico \\
\hline M1Fuji II LC & $2,80 \bullet \bullet$ & $1,26 \bullet$ & $1,57 \mathbf{\Delta}$ \\
\hline M2 KetacN100 & $0,93 \bullet$ & 3,79 & $1,42 \downarrow \Delta$ \\
\hline
\end{tabular}

Valor crítico de Tukey: 1,45

$\mathrm{Dp} \pm 0,22$

Tabla 6. Medias de Alteración de color $(\Delta E)$ para la interacción Solución x Tiempo. 


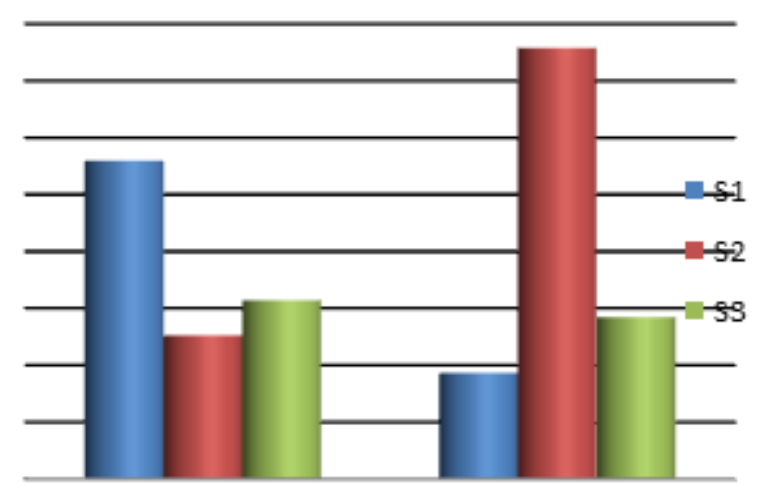

Gráfico 4. Medias de $\Delta$ E para la Interacción Solución $x$ Material. (Sentido de las líneas).

cial $(\mu \mathrm{m})$, cuando fue analizado aisladamente. Las medias para este factor constan en la Tabla 8 y Gráfico 5, a continuación

\section{Factor Material}

El factor Material, cuando fue analizado aisladamente, mostró tener efecto estadísticamente significativo sobre la rugosidad superficial. En la tabla 9 se observa que el Material Fuji II LC®

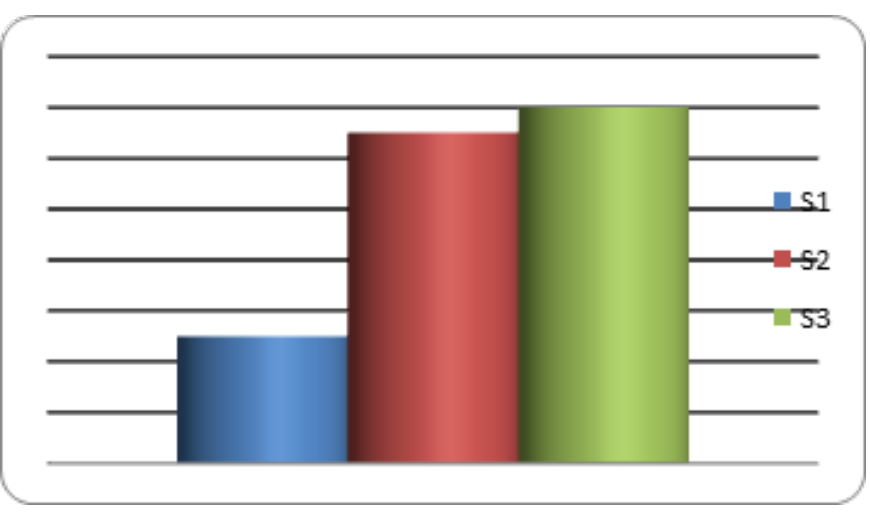

Gráfico 5. Medias de Rugosidad superficial $(\mu \mathrm{m})$ para el factor Solución.

(M1) determinó menor media de rugosidad superficial que el Material Ketac N100® (M2). Gráfico 6.

\section{Factor Tiempo}

El tiempo fue el factor que, siendo analizado aisladamente, demostró tener un efecto estadísticamente significante sobre la rugosidad superficial. En la Tabla 10 y el gráfico 7 se ob-

\begin{tabular}{|c|c|c|c|c|c|}
\hline Fonte de Variaçäo & Soma de Quadr. & G.L. & $\begin{array}{l}\text { Quadr. } \\
\text { Médios }\end{array}$ & (F) & Prob.(H0) \\
\hline Factor SOLUCIÓN (S) & 0.2231 & 2 & 0.1115 & 2.07 & $13.2652 \% \mathrm{~ns}$ \\
\hline Factor MATERIAL (M) & 3.2821 & 1 & 3.2821 & 60.83 & $0.0000 \%^{*}$ \\
\hline InteracciónMxS & 0.4822 & 2 & 0.2411 & 4.47 & $1.4900 \%^{* *}$ \\
\hline Resíduo I & 3.5608 & 66 & 0.0540 & & \\
\hline Factor TIEMPO (T) & 0.1407 & 1 & 0.1407 & 7.81 & $0.6831 \%^{*}$ \\
\hline InteracciónTxS & 0.0772 & 2 & 0.0386 & 2.14 & $12.3260 \% \mathrm{~ns}$ \\
\hline Interacción TxM & 0.0544 & 1 & 0.0544 & 3.02 & $8.3018 \% \mathrm{~ns}$ \\
\hline Resíduo II & 1.1886 & 66 & 0.0180 & & \\
\hline Variaçäo total & 9.2549 & 143 & & & \\
\hline
\end{tabular}

Tabla 7. Resumen del análisis de varianza: Valores de Rugosidad superficial ( $\mu \mathrm{m})$. 


\begin{tabular}{|c|c|c|}
\hline $\begin{array}{c}\text { S1 } \\
\text { Agua destilada }\end{array}$ & $\begin{array}{c}\text { S2 } \\
\text { Bebida } \\
\text { Carbonatada }\end{array}$ & $\begin{array}{c}\text { S3 } \\
\text { Desafío Cítrico }\end{array}$ \\
\hline 0.63 & 0.71 & 0.72 \\
\hline
\end{tabular}

Ns

$\mathrm{Dp} \pm 0,03$

Tabla 8. Medias de rugosidad superficial $(\mu \mathrm{m})$ para el Factor Solución.

serva la media obtenida en el tiempo de 14 días (T1) que fue menor al obtenido en el tiempo inicial (T0).

\section{Interacción Material x Solución}

En la tabla 11 constan las medias de Rugosidad Superficial para la interacción Material x Solución. El análisis realizado demostró que la interacción entre estos factores fue estadísticamente significante. Lo que justifica su análisis, ya que el factor Solución no fue significante cuando analizado aisladamente. Puede verificarse que para el Material Fuji II LC® (M1), las medias determinadas por cada una de las soluciones fueron estadísticamente iguales. Y para el Ketac N100® (M2), la Solución Desafío cítrico (S3) determinó mayor media de rugosidad superficial que la determinada por el grupo control - Agua desti-

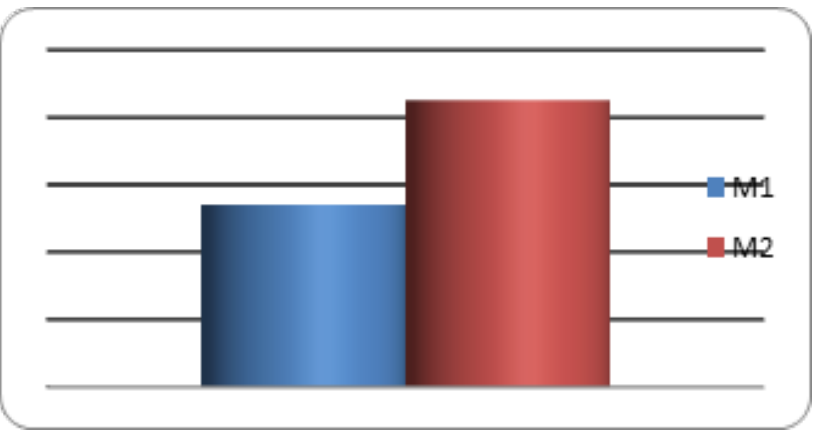

Gráfico 6. Medias de Rugosidad superficial ( $\mu \mathrm{m})$ para el factor Material.

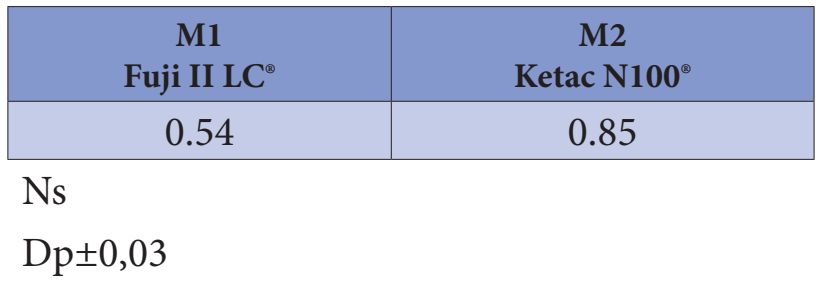

Tabla 9. Medias de rugosidad superficial $(\mu \mathrm{m})$ para el Factor Material.

lada (S1). Entre tanto, la Bebida carbonatada (S2) quedó en posición intermedia. Cuando se analiza en el sentido de las columnas, se constata que el Material Fuji II LC® (M1) presentó medias de rugosidad superficial $(\mu \mathrm{m})$ menores que las presentadas por Ketac N100® (M2), cuando son sometidos a las Soluciones Bebida carbonatada (S2) y al Desafío cítrico (S3). No hubo diferencia entre los materiales para la Solución Agua destilada (S1). Esto puede ser visto en el Gráfico 8 y 9.

\section{Interacción Solución x Tiempo}

Se organizó la Tabla 12 y el Gráfico 10 con las medias para la Interacción Solución x Tiempo. La relevancia de esta interacción está en poder demostrar su no significancia estadística, pues permitió verificar que cuando asociamos el factor Tiempo al factor Solución, o sea, cuando estos dos factores interactúan no determina un efecto significante en la variable rugosidad superficial. Esto principalmente considerando el período de tiempo determinado para este estudio.

\begin{tabular}{|c|c|}
\hline Inicial (T0) & 14 días (T1) \\
\hline 0.72 & 0,66 \\
\hline
\end{tabular}

Valor crítico de Tukey: 0,04

$\mathrm{Dp} \pm 0.01$

Tabla 10. Medias de rugosidad superficial $(\mu \mathrm{m})$ para el factor Tiempo. 


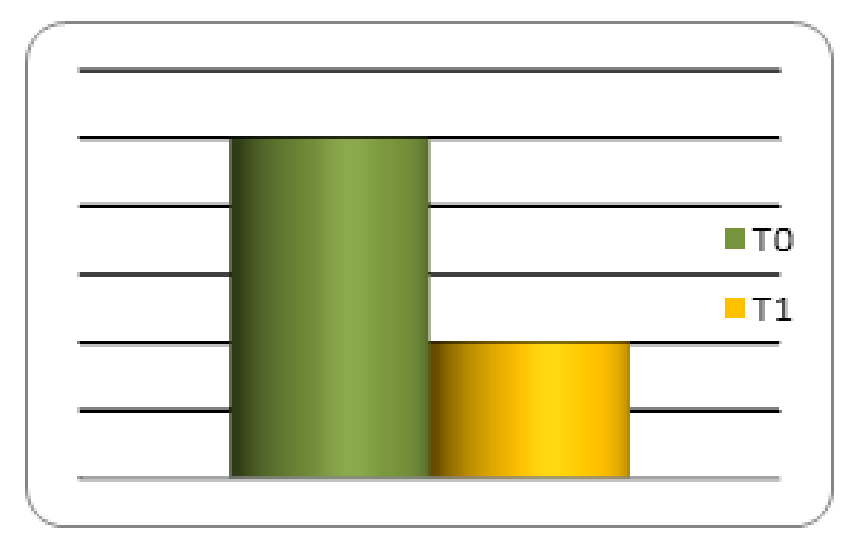

Gráfico 7. Medias de Rugosidad superficial ( $\mu m)$ para el factor Tiempo.

Interacción Material x Tiempo.

La interacción Tiempo x Material tampoco fue significante estadísticamente (Tabla13). Para los cementos ionómericos estudiados asociados con el factor Tiempo no determinó alteraciones estadísticamente significantes sobre la rugosidad superficial. Gráfico 11.

\section{DISCUSIÓN}

Actualmente, sabemos que los ionómeros vítreos son muy utilizados en odontopediatría, principalmente, por sus propiedades de adherencia a la estructura dental, liberación de flúor y su propiedad estética. Son considerados como

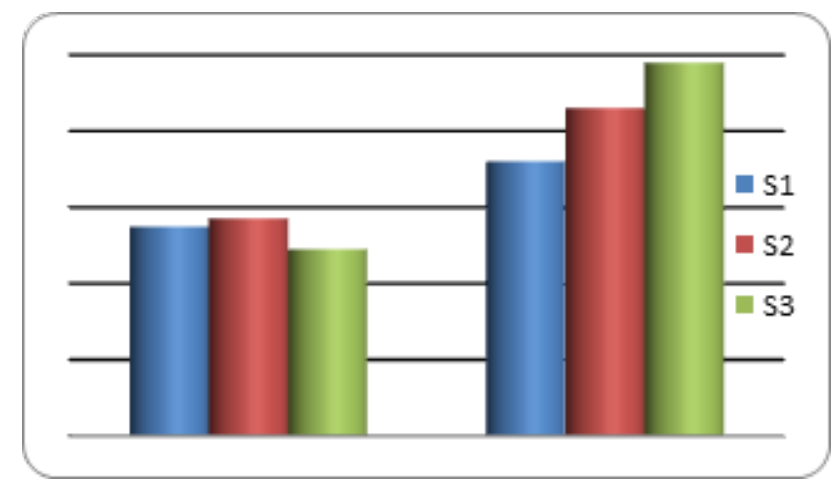

Gráfico 8. Medias de rugosidad superficial $(\mu \mathrm{m})$ para la Interacción Material x Solución (Sentido de las líneas).

\begin{tabular}{|l|c|c|c|}
\hline & $\begin{array}{c}\text { S1 } \\
\text { Aguadestilada }\end{array}$ & $\begin{array}{c}\text { S2 } \\
\text { Bebida } \\
\text { Carbonatada }\end{array}$ & $\begin{array}{c}\text { S3 } \\
\text { Desafío } \\
\text { Cítrico }\end{array}$ \\
\hline Fuji II LC & $0.55 \boldsymbol{\Delta}$ & $0.57 \boldsymbol{\Delta}$ & $0.49 \boldsymbol{\Delta}$ \\
\hline KetacN100 $^{\infty}$ & $0.72 \bullet$ & $0.86 \bullet \bullet$ & $0.98 \boldsymbol{}$ \\
\hline
\end{tabular}

Valor crítico de Turkey: 0.19

$\mathrm{Dp} \pm 0.04$

Tabla 11. Medias para Rugosidad Superficial ( $\mu \mathrm{m})$ para la interacción Material x Solución.

buenas alternativas a la amalgama y otro tipo de restauraciones antiguamente utilizadas en la odontología infantil. Sin embargo, estas ventajas antagonizan con su estabilidad de color y sus bajas propiedades mecánicas. ${ }^{17}$

Por otra parte, consideremos que los materiales dentales utilizados son sometidos, en el medio oral, a condiciones clínicas rigurosas como alteración del $\mathrm{pH}$, del flujo salivar y de temperatura, entre otros. El rendimiento clínico de las restauraciones dentales puede verse afectado por los cambios de $\mathrm{pH}$ en la cavidad oral. Debido al aumento en el consumo de bebidas de bajo $\mathrm{pH}$, las superficies de los materiales pueden llegar a ser ásperas y sin brillo a un nivel clínicamente detectable. ${ }^{18}$

Para simular estas condiciones, a los que los materiales estéticos son sometidos en la cavi-

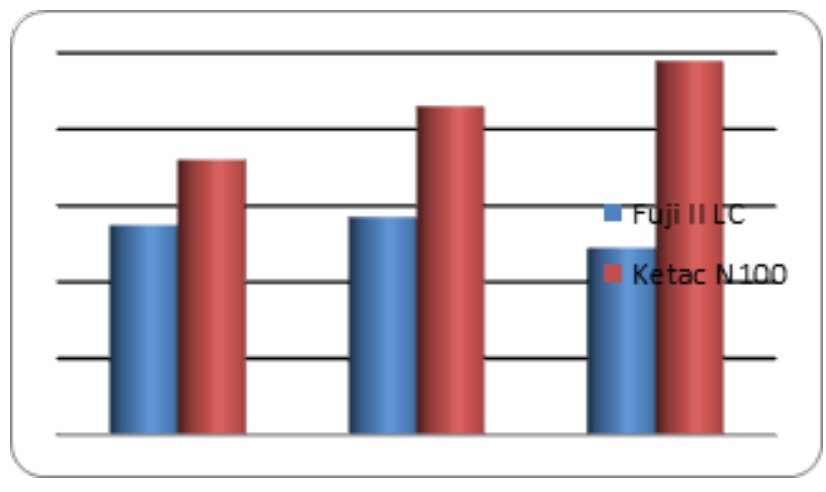

Gráfico 9. Medias de rugosidad superficial ( $\mu \mathrm{m})$ para la Interacción Solución x Material (Sentido de las columnas). 


\begin{tabular}{|l|c|c|}
\hline & Inicial (T0) & 14 días (T1) \\
\hline S1 - Agua destilada & 0.63 & 0.63 \\
\hline S2 -Bebida Carbonatada & 0.65 & 0.68 \\
\hline S3 -Desafío Citrico & 0.78 & 0.70 \\
\hline
\end{tabular}

Ns

$\mathrm{Dp} \pm 0.02$

Tabla 12. Medias de Rugosidad Superficial $(\mu m)$ para la interacción Solución x Tiempo.

dad bucal, la literatura demuestra la existencia de diferentes métodos de verificación del comportamiento de los mismos en un período de tiempo que simulan su envejecimiento. De entre éstos, podemos mencionar que, se destacan los métodos de inmersión en soluciones. ${ }^{19,20}$

Basándonos en estos aspectos se determinó realizar este estudio in vitro, donde se han fabricado los cuerpos de prueba y se los ha sometido a diferentes soluciones para estudiar el efecto causado por la ingestión de bebidas consumidas más frecuentemente por los niños en las restauraciones dentales realizadas con dos tipos de cementos de ionómeros vítreos.

En este estudio, se decidió evaluar la rugosidad superficial y la alteración de color que producen tres tipos diferentes de bebidas ${ }^{21}$ en las restauraciones dentales, aproximándonos a la frecuencia en la ingestión que realizarán los niños por un período determinado de tiempo. Para tratar de direccionar al odontopediatra de las posibles

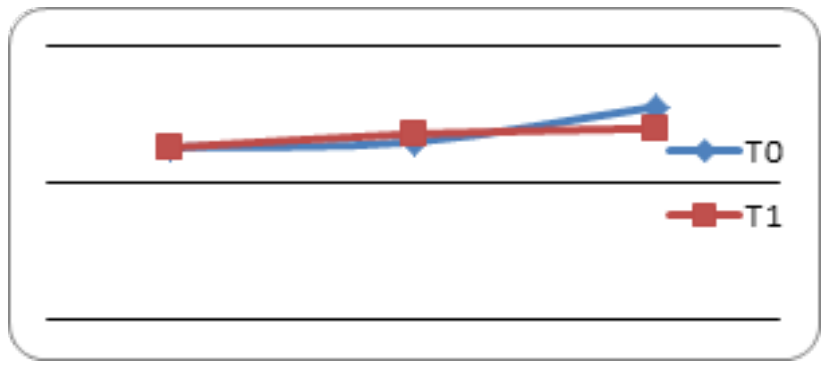

Gráfico 10. Medias de Rugosidad Superficial ( $\mu \mathrm{m})$ para la interacción Solución $x$ Tiempo. complicaciones que pudieran surgir con las restauraciones realizadas.

La alteración de color en los materiales restauradores estéticos se han atribuido a una amplia variedad de posibles causas. El desgaste o degradación química puede aumentar la susceptibilidad de estos materiales a las manchas extrínsecas. Otros factores causantes que pueden contribuir al cambio en el color de los materiales de restauración estéticos incluyen: acumulación de manchas, deshidratación, absorción de agua, filtración, falta de unión y rugosidad superficial. ${ }^{22}$

Para un mejor análisis de los resultados del cambio de color $\left(\Delta \mathrm{E}^{*}\right)$, es importante tener en cuenta que, de acuerdo con el sistema CIE L*a*b*, las alteraciones de color entre 0.0 y 0.5 son consideradas como trazas o vestigios, de 0,5 a 1,5 como superficiales y de 1,5 a 3,0 como perceptibles ${ }^{23}$ y los valores superiores a 3,3 son clínicamente inaceptables.

Los resultados obtenidos de alteración de color en el presente estudio demuestran que el factor solución tuvo un efecto estadísticamente significante, pues la bebida carbonatada produjo mayor grado de alteración de color que la solución placebo (agua destilada) y el jugo cítrico. Según los conceptos expuestos anteriormente, en relación a los valores medios del $\Delta \mathrm{E}^{*}$ para el factor solución, se sugiere que las alteraciones de color son clínicamente inaceptables. El hecho que la bebida carbonatada produce un oscurecimiento importante en los cementos de ionómeros vítre- 
os modificados con resinas en todas sus formas está de acuerdo con el estudio in vitro realizado por Mohan y colaboradores $(2008)^{20}$ donde se recomienda evitar el uso de estos materiales en el sector anterior debido a su alto índice de comprometimiento estético.

Con relación al factor material, se pudo verificar que no hubo un efecto significante estadísticamente sobre la alteración de color. Estudios de Yap y colaboradores (1999), ${ }^{24}$ ya determinaron que los cementos de ionómeros vítreos modificados con resina sufren cambios de color durante el proceso de fotopolimerización. Este cambio de color es atribuido a que la reacción ácido-base producida es retardada por el proceso de fotopolimerización. Sin embargo, debido a que ambos materiales son cementos de ionómeros modificados con resinas, no han sido encontrados en la literatura trabajos con una metodología semejante que permita una discusión directa de los resultados obtenidos.

En este estudio, se verificó un efecto significante para la interacción material x solución, ya que al evaluar ambos materiales (Fuji IILC®) y Ketac $\mathrm{N} 100 \AA)$ luego de ser sometidos a las tres soluciones, se encontró en el Fuji II LC una menor alteración de color con relación al Ketac N100 luego de ser sometidos a una bebida carbonatada (S2). Esto, concuerda con otros estudios como el de Bagheri y colaboradores (2004) $)^{25}$ que han concluído que los compómeros y los ionómeros vítreos modificados con resinas son susceptibles a la coloración después de ser sometidos a diversas soluciones y luego de haber trascurrido un período de tiempo. También los análisis de Mohan y colaboradores $(2008)^{20}$ demostraron que la solución carbonatada, específicamente, compromete de manera significativa la estabilidad de color en los cementos ionómeros modificados con resinas luego de 72 horas de exposición a la solución.

Con relación a la rugosidad superficial, mucho se ha investigado en relación de resinas compuestas y bebidas colorantes ${ }^{15,26}$ pero existen pocos estudios que evalúan estos dos materiales mencionados anteriormente sometidos a tres bebidas colorantes, por lo cual se determinó realizar este trabajo teniendo en cuenta aquellos que son más frecuentemente ingeridos por los niños en nuestro país.

Con respecto al factor solución, este estudio nos permite concluir que el mismo no tiene un valor estadísticamente significativo en la rugosidad superficial, y esto no está de acuerdo con el trabajo de Badra et al. (2005) ${ }^{27}$ donde afirma que la rugosidad superficial está directamente relacionada con la acción de algunos productos utilizados en la cavidad bucal aunque el estudio se ha realizado en resinas compuestas y resinas fluídas. Abubakr y colaboradores $(2000)^{28}$ evaluaron tres tipos de propiedades mecánicas encontrando en todas ellas una diferencia estadísticamente significante siendo sometidas a las soluciones carbonatadas y jugo cítrico y concluyeron que éstas afectan la fuerza compresiva, la microdureza, la solubilidad y la textura superficial de los materiales de restauración; cabe acotar que fueron observaciones realizadas hasta los 60 días de inmersión.

Con relación al factor material, en el presente estudio se encontró una diferencia estadísticamente significativa entre el Fuji II LC® y el Ketac N 100®, presentando este último un incremento en la rugosidad superficial. Esta conclusión no concuerda con el estudio realizado por Briso et al. $(2011)^{29}$ donde si se observó un incremento con el Fuji II LC® aunque éste es evaluado junto con otros ionómeros modificados con resinas y otras dos resinas compuestas. Resaltamos que, en 
este mismo estudio, se relata que varios factores pueden afectar los resultados como son la manipulación y la composición de estos materiales.

Con relación al factor tiempo, se encontró un efecto significante considerando el tiempo inicial (T0) y a los 14 días el tiempo final (T1). Se asume que nuevos estudios deberían ser realizados para un período mayor de tiempo de observación y lectura de estas propiedades a ser evaluadas, pudiendo allí encontrar alguna variación que pudieran interferir en la rugosidad superficial. ${ }^{13}$ Muchos autores, relatan que la rugosidad superficial de los materiales es el resultado de las interacciones de muchos factores asociados con tipo, tamaño y distribución de partículas, grado de polimerización, así como el eficiente contacto entre las partículas y la matriz.

En cuanto a las interacciones entre los factores, material x solución fue la que tuvo un efecto significativo que demostró mayor media de rugosidad del Ketac N100® sometido a la solución - desafío cítrico. Estudios de Hamouda y colaboradores $(2011)^{18}$ establecen la actividad erosiva de los ácidos cítricos, maléicos, fosfóricos entre otros contenidos en las bebidas aumentan significativamente la rugosidad superficial de los cementos ionomeros modificados.

Se sabe que los estudios tanto in vitro como aquellos in situ presentan limitaciones en cuanto a evaluar y medir el comportamiento de los materiales de restauración, ya que en la cavidad bucal, éstos se encuentran sometidos a factores individuales como ser biofilm, calidad y periodicidad de la higiene y acción mecánica del todo el sistema oral, lo cual tiene acción directa sobre el comportamiento físico-mecánico de los materiales odontológicos.

Dos aspectos a considerar, con los resultados analizados, son importantes; por un lado, he- mos encontrado que la rugosidad superficial del Ketac N100® es significativamente mayor al Fuji II LC® cuando es analizado aisladamente y en cuanto es observado en interacción con las soluciones y por otra parte, que de las tres soluciones en estudio fue la bebida carbonatada la que produjo mayor alteración de color. Adicionando a esto que con los resultados analizados hemos investigado el comportamiento físicomecánico de los cementos de ionómeros vítreos modificados con resinas y los recientes nanoionómeros incorporados a la práctica colaborando así a que el profesional, que trata al infante, considere estos aspectos para poder realizar en su práctica privada restauraciones y tratamientos dentro de los límites clínicamente aceptables, sin riesgo de causar alteraciones perceptibles en dichos tratamientos.

\section{CONCLUSIONES}

De acuerdo con la metodología utilizada se puede concluir:

- Hubo una mayor alteración de color del Ketac N100® luego de ser sumergido a la bebida carbonatada.

- Para el parámetro $\Delta \mathrm{E}^{*}$ la solución carbonatada detecta mayor alteración de color.

- Los materiales estudiados no influenciaron el comportamiento de la estabilidad del color, analizándolos aisladamente.

- La inmersión durante 14 días en diferentes bebidas influenció la rugosidad superficial.

- $\quad$ El Ketac N 100® detectó mayor rugosidad superficial que el Fuji II LC®.

- El desafio cítrico aumenta significativamente la rugosidad superficial del KetacN100®. 


\section{Referencias bibliográficas}

1. Ozdemir-Ozenen D, Sungurtekin E, Issever H, Sandalli N. Surface roughness of fluoride-releasing restorative materials after topical fluoride application. Eur Arch Paediatr Dent. 2013;14(1):68-72.

2. Bala O, Arisu HD, Yikilgan I, Arslan S, Gullu A. Evaluation of surface roughness and hardness of different glass ionomer cements. Eur J Dent. 2012; 6(1):79-86.

3. Nakajo K, Imazato S, Takahashi Y, Kiba W, Ebisu S, Takahashi N. Fluoride released from glass-ionomer cement is responsible to inhibit the acid production of caries-related oral streptococci. Dent Mater. 2009; 25(6): 703-8.

4. Ling L, Xu X, Choi GY, Billodeaux D, Guo G, Diwan RM. Novel F-releasing composite with improved mechanical properties. J Dent Res. 2009 1;88(1):83-8.

5. Anusavice KJ, Zhang NZ, Shen C. Controlled release of chlorhexidine from UDMA-TEGDMA resin. J Dent Res. 2006 Oct 1;85(10):950-4.

6. Wiegand A, Buchalla W, Attin T. Review on fluoride-releasing restorative materials-fluoride release and uptake characteristics, antibacterial activity and influence on caries formation. Dent Mater. 2007 Mar 31;23(3):343-62.

7. Coutinho E, Cardoso MV, De Munck J, Neves AA, Van Landuyt KL, Poitevin A, Peumans M, Lambrechts P, Van Meerbeek

B. Bonding effectiveness and interfacial characterization of a nano-filled resin-modified glass-ionomer. Dent Mater. 2009; 25(11): 1347-57.

8. Peumans M, Kanumilli P, De Munck J, Van Landuyt K, Lambrechts P, Van Meerbeek B. Clinical effectiveness of contemporary adhesives: a systematic review of current clinical trials. Dent Mater. 2005; 21(9): 864-81.

9. Xu HH, Moreau JL, Sun L, Chow LC. Novel CaF2 nanocomposite with high strength and fluoride ion release. J Dent Res. 2010; 89(7): 739-45.

10. Mitra SB, Wu D, Holmes BN. An application of nanotechnology in advanced dental materials. JADA. 2003; 134(10): 1382-90.

11. Korkmaz Y, Gurgan S, Firat E, Nathanson D. Shear bond strength of three different nano-restorative materials to dentin. Oper Dent. 2010 Jan;35(1):50-7.

12. Carvalho FG, Sampaio CS, Fucio SB, Carlo HL, Correr-Sobrinho L, Puppin-Rontani RM. Effect of chemical and mechanical degradation on surface roughness of three glass ionomers and a nanofilled resin composite. Oper Dent. 2012; 37(5): 509-17. 13. De Oliveira AL, Garcia PP, Santos PA, Campos JÁ. Surface roughness and hardness of a composite resin: influence of finishing and polishing and immersion methods. Mat Res. 2010 Sep;13(3):409-15.

14. Dorini, A.L. Avaliação da pigmentação superficial por café e vino em compositos odontologicos. Faculdade de Odontologia de Piracicaba. Universidad Estadual de Campinas. 2001. Tesis doctoral en Clinica Odontologica. Disponible en internet: $<$ http://libdigi.unicamp.br/document/?code=vtls000231852

15. Yousef M, Abo El Naga A. Color stability of different restoratives after exposure to coloring agents. J Am Sci. 2012;8:20-6.

16. Maneenut C, Sakoolnamarka R, Tyas MJ. The repair potential of resin-modified glass-ionomer cements. Dent Mater. 2010 Jul 31;26(7):659-65.

17. Sidhu, S. K. Clinical evaluations of resin-modified glass-ionomer restorations. Dent Mater 2010; 26 (1): 7-12.

18. Hamouda IM. Effects of various beverages on hardness, roughness, and solubility of esthetic restorative materials. Journal of Esthetic and Restorative Dentistry. 2011; 23(5): 315-22.

19. Lee YK, El Zawahry M, Noaman KM, Powers JM. Effect of mouthwash and accelerated aging on the color stability of esthetic restorative materials. Am J Dent. 2000; 13(3): 159-61.

20. Mohan M, Shey Z, Vaidyanathan J, Vaidyanathan TK, Munisamy S, Janal M. Color changes of restorative materials exposed in vitro to cola beverage. Ped Dent. 2008; 30(4): 309-16. 
21. Lussi A, Kohler N, Zero D, Schaffner M, Megert B. A comparison of the erosive potential of different beverages in primary and permanent teeth using an in vitro model. Eur J Oral Sci. 2000; 108(2): 110-4.

22. Ahmed KI, Sajjan G. Color stability of ionomer and resin composite restoratives in various environmental solutions: An invitro reflection spectrophotometric study. J Conserv Dent. 2005; 8(1): 45.

23. Noie F, O'Keefe KL, Powers JM. Color stability of resin cements after accelerated aging. Int J Prosthodont. 1995; 8(1): 51-5.

24. Yap AU, Sim CP, Loganathan V. Color stability of a resin-modified glass ionomer cement. Oper Dent. 2001; 26(6): 591-6.

25. Bagheri R, Burrow MF, Tyas M. Influence of food-simulating solutions and surface finish on susceptibility to staining of aesthetic restorative materials. Journal of Dentistry. 2005; 33(5): 389-98.

26. Godoi AP. Efeito in situ da associação de chá preto ao uso diário de clorexidina na alteração de cor e rugosidade superficial de resinas compostas (Doctoral dissertation, Universidade de São Paulo).

27. Badra VV, Faraoni JJ, Ramos RP, Palma-Dibb RG. Influence of different beverages on the microhardness and surface roughness of resin composites. Oper Dent. 2005; 30(2): 213-9.

28. Abu-bakr N, Han L, Okamoto A, Iwaku M. Changes in the mechanical properties and surface texture of compomer immersed in various media. J Prosthet Dent. 2000; 84(4): 444-52.

29. Briso AL, Caruzo LP, Guedes AP, Catelan A, Santos PD. In vitro evaluation of surface roughness and microhardness of restorative materials submitted to erosive challenges. Oper Dent. 2011; 36(4): 397-402.

Recibido: 09/06/2016

Aceptado 12/04/2017

Correspondencia: Marisol Carrollo Tabakman mscarrillod@hotmail.com 